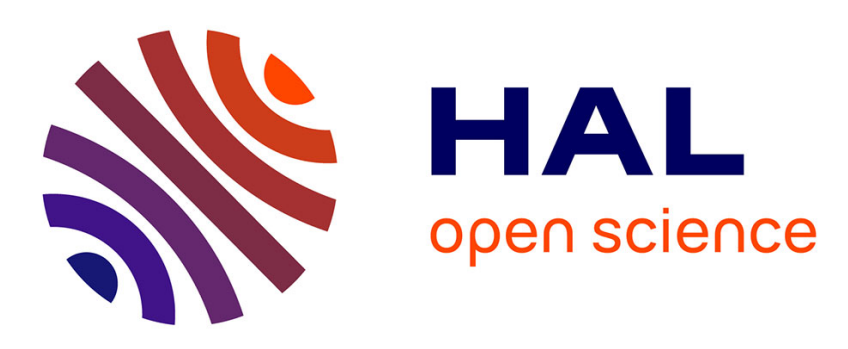

\title{
Evaluating signs of determinants using single-precision arithmeti
}

\author{
Francis Avnaim, Jean-Daniel Boissonnat, Olivier Devillers, Franco P. \\ Preparata, Mariette Yvinec
}

\section{To cite this version:}

Francis Avnaim, Jean-Daniel Boissonnat, Olivier Devillers, Franco P. Preparata, Mariette Yvinec. Evaluating signs of determinants using single-precision arithmeti. Algorithmica, 1997, 17 (2), pp.111132. 10.1007/BF02522822 . inria-00090613

\section{HAL Id: inria-00090613 https://hal.inria.fr/inria-00090613}

Submitted on 1 Sep 2006

HAL is a multi-disciplinary open access archive for the deposit and dissemination of scientific research documents, whether they are published or not. The documents may come from teaching and research institutions in France or abroad, or from public or private research centers.
L'archive ouverte pluridisciplinaire HAL, est destinée au dépôt et à la diffusion de documents scientifiques de niveau recherche, publiés ou non, émanant des établissements d'enseignement et de recherche français ou étrangers, des laboratoires publics ou privés. 


\title{
Evaluating signs of determinants using single-precision arithmetic *
}

\author{
Francis Avnaim $^{\dagger} \quad$ Jean-Daniel Boissonnat ${ }^{\ddagger} \quad$ Olivier Devillers ${ }^{\S}$ \\ Franco P. Preparata ${ }^{\pi} \quad$ Mariette Yvinec ${ }^{\|}$ \\ Revised version
}

\begin{abstract}
We propose a method to evaluate signs of $2 \times 2$ and $3 \times 3$ determinants with $b$-bit integer entries using only $b$ and $(b+1)$-bit arithmetic respectively. This algorithm has numerous applications in geometric computation and provides a general and practical approach to robustness. The algorithm has been implemented and compared with other exact computation methods.

Keywords : computational geometry, exact arithmetic, precision, robust algorithms.

\section{Introduction}

Most decisions in geometric algorithms are based on signs of determinants. For example, deciding if a point belongs to a given halfspace or a given ball reduces to evaluating the sign of a determinant. Moreover, such evaluations are often the only numerical parts of the entire algorithm. Therefore, it is crucial to have reliable answers to such tests.

This observation relates to the very model of Computational Geometry, whose assumption is that geometric parameters are real numbers and that arithmetic operations are performed with infinite precision.
\end{abstract}

\footnotetext{
* This work was partially supported by the ESPRIT Basic Research Action 7141 (ALCOMII) and by NSF Grant CCR 91-96176. Part of this work was done while J.-D. Boissonnat was visiting Brown University.

†INRIA, BP93, 06902 Sophia-Antipolis cedex (France),

${ }^{\ddagger}$ INRIA, E-mail: Jean-Daniel.Boissonnat@sophia.inria.fr.

$\S$ INRIA, E-mail: Olivier.Devillers@sophia.inria.fr.

"Brown University, Department of Computer Science, Providence, RI 02912-1910 (USA) E-mail: franco@cs.brown.edu.

"INRIA and CNRS, URA 1376, Laboratoire I3S, 250 Rue Albert Einstein, 06560 Valbonne (France), E-mail: Mariette.Yvinec@sophia.inria.fr.
} 
Obviously, such assumption does not hold when algorithms are translated into computer programs, where the parameters are represented either as integers or as floating-point numbers. Floating-point implementations, although naively a reasonable approach to real-number arithmetic, have been shown to have serious shortcomings, since they may cause not only numerical errors but also fatal membership errors (such as inclusion of a point in an interval to which it does not belong, etc.).

This difficulty has received some deserved attention in recent years (see, e.g., [For89, GY86, HHK89, Mil88a, Mil88b, Mil89, GSS89, SI88]) and several approaches have been proposed on how to obviate the shortcoming. The common objective is to produce robust algorithms, namely algorithms whose answer is a (small) perturbation of the correct answer (as produced by the infinite-precision algorithm). As noted by Fortune [For89], there are basically two categories of approaches to this objective: The most common one resorts to approximate (i.e., rounded) computations, and uses properties of the assumed primitives to establish the topological correctness of the results (i.e., robustness) (see, e.g., [For89, For92, FM91, HHK89, GSS89]). The other uses exact (i.e., integer) computations, but, since multiprecision integer arithmetic is required ( $d$-fold for a dimension $d$ determinant), a straightforward implementation of this approach has a large performance penalty (see [FV93] for a detailed analysis). Significant improvements over the naive method have been recently obtained. In particular, a promissing approach consists in combining multiprecision integer (or rational) arithmetic and a floating point filter based on interval analysis [KLN91, FV93, BJMM93].

Our approach falls in the exact integer arithmetic category and our objective is to use as few bits as possible to evaluate signs of determinants. Typically, we will use no more bits than the number $b$ of bits used to code the entries. This implies that we would not do multiplications of the entries and, a fortiori, we would not compute any determinants when we evaluate the signs.

To the best of our knowledge, the only related attempt has been made by Clarkson [Cla92]. Clarkson uses an adaptation of the Gram-Schmidt procedure for computing an orthogonal basis, and employs approximate arithmetic. For a $d \times d$ determinant with $b$-bit integer entries, Clarkson's algorithm runs in time $O\left(d^{3} b\right)$ and uses $2 b+1.5 d$ bits to represent the values. Our algorithm is quite different. It is limited to $2 \times 2$ and $3 \times 3$ determinants and its asymptotic worst-case complexity is worse than that of Clarkson. However, it is simpler, it uses respectively $b$ and $(b+1)$-bit representations, and extensive simulations have shown that it performs well in practice. Since the most common applications are two- and three-dimensional, our method is competitive in this range of parameters.

The paper is organized as follows. In Section 2, we shall discuss the two-dimensional case in detail, both because it provides insights for the three-dimensional case and because it is used to resolve the determination of the sign of a three-dimensional determinant in Section 
3. In Section 4, we present some significant applications of the outlined technique in computational geometry. In Section 5, we present and discuss experimental results and compare our algorithm with the straightforward computation using floating point arithmetic or other exact arithmetics.

\section{Two-dimensional case}

\subsection{The algorithm}

Let $D=\left|\begin{array}{ll}x_{1} & y_{1} \\ x_{2} & y_{2}\end{array}\right|$ be a $2 \times 2$ determinant whose entries are $b$-bit integers.

If one of the four entries is zero, $D$ reduces to the product of two integers and the sign of $D$ follows from the sign of the two integers. In the rest of this section, we assume that all four entries are nonzero.

Without loss of generality, we may assume that all the entries are strictly positive. Indeed, if an odd number of entries are negative, then the sign of $D$ is trivially obtained. If two entries are negative, either they belong to the same row or the same column, in which case changing their sign changes the sign of $D$, or they belong to a diagonal, in which case changing their sign does not change $D$. If all entries are negative, changing their sign does not change $D$.

Furthermore, we may assume without loss of generality that $x_{2} \geq$ $x_{1}$ and $y_{2} \geq y_{1}$. The case where $x_{2}<x_{1}$ and $y_{2}<y_{1}$ can be transformed to the previous one by exchanging the two rows of the matrix, which yields a change of the sign of $D$. In the other cases, the sign of $D$ can be obtained readily; specifically, if $x_{1} \leq x_{2}$ and $y_{2}<y_{1}, D<0$, and, if $x_{1}>x_{2}$ and $y_{2} \geq y_{1}, D>0$.

Under the above assumptions, we can write:

$$
x_{2}=x_{1} k_{1}+x_{r} \text { with } k_{1} \in \mathbb{N} \text { and } 0 \leq x_{r}<x_{1}
$$

and define:

$$
y_{r}=y_{2}-k_{1} y_{1}
$$

Then

$$
D=\left|\begin{array}{ll}
x_{1} & y_{1} \\
x_{2} & y_{2}
\end{array}\right|=\left|\begin{array}{cc}
x_{1} & y_{1} \\
x_{2}-k_{1} x_{1} & y_{2}-k_{1} y_{1}
\end{array}\right|=\left|\begin{array}{cc}
x_{1} & y_{1} \\
x_{r} & y_{r}
\end{array}\right|
$$

If $y_{1}>\frac{2^{b}}{k_{1}}$ then $y_{r}$ cannot be computed, but in that case $y_{r}$ is certainly negative and thus $D<0$ otherwise $y_{r}$ can be computed. If it is outside the range $\left[0, y_{1}\right]$ the sign of $D$ can be obtained too: if $y_{r}<0$ then $D<0$ and if $y_{r}>y_{1}$ then $D>0$. Moreover, if $x_{r}<\frac{x_{1}}{2}$ and $y_{r}>\frac{y_{1}}{2}$, then $D>0$, and if $x_{r}>\frac{x_{1}}{2}$ and $y_{r}<\frac{y_{1}}{2}$, then $D<0$. Otherwise we rewrite $D$ as follows:

$$
\begin{aligned}
& \text { if } x_{r}<\frac{x_{1}}{2} \text { and } y_{r}<\frac{y_{1}}{2} \text { then } D=\left|\begin{array}{cc}
x_{1} & y_{1} \\
x_{r} & y_{r}
\end{array}\right| \\
& \text { if } x_{r}>\frac{x_{1}}{2} \text { and } y_{r}>\frac{y_{1}}{2} \text { then } \quad D=\left|\begin{array}{cc}
x_{1} & y_{1} \\
x_{1}-x_{r} & y_{1}-y_{r}
\end{array}\right|
\end{aligned}
$$


In both cases, we get a new determinant where both entries of the second row have been divided by at least two, and we can iterate the computation.

In conclusion, at each iteration, using only comparisons and euclidean divisions, either the algorithm stops or it iterates on a reduced problem, where a row is replaced by one whose entries are less than half the size of the original ones. Hence, the number of iterations is bounded from above by the logarithm of the largest representable integer, i.e. the number $b$ of bits in the binary representation of the initial entries.

We sum up the results in the following theorem.

Theorem 1 Let $D$ be $a 2 \times 2$ determinant with b-bit integer entries. There exists an algorithm that evaluates the sign of $D$ using only b-bit arithmetic. The algorithm requires at most $b$ iterations, each iteration involving $O(1)$ additions/subtractions, comparisons and euclidean divisions.

\section{Three dimensions}

\subsection{Geometric intuition}

Let $D=\left|\begin{array}{lll}x_{1} & y_{1} & z_{1} \\ x_{2} & y_{2} & z_{2} \\ x_{3} & y_{3} & z_{3}\end{array}\right|$ be a $3 \times 3$ determinant with $b$-bit integer entries. Vector $\left(x_{i}, y_{i}, z_{i}\right)$ is denoted $U_{i}$ and the unit vectors along the three axis will be denoted $E_{x}, E_{y}$ and $E_{z}$. The $z$ direction is called vertical and $u$ denotes the vertical projection of vector $U$ onto the horizontal plane $z=0$. For convenience, we will often identify a vector $U$ with the point whose coordinate vector is $U$. Without loss of generality, we can assume that the $z_{i}$ are nonnegative and that $z_{3} \geq z_{1}, z_{2}$.

The basic idea is as follows : unless the sign of $D$ can be directly assessed, we replace the original matrix with a matrix having the same determinant, but provably smaller entries. This assures that the evaluation will terminate. The basic device used is the standard addition to a row of a linear combination of the other rows.

More specifically, assume (here and in Sections 3.1-3.6), that the projections $u_{1}, u_{2}$ of $U_{1}, U_{2}$ are noncolinear (i.e. independent) vectors. This implies that the three vectors $U_{1}, U_{2}$ and $E_{z}$ are linearly independent, and we can express $U_{3}$ as

$$
U_{3}=\kappa_{1} U_{1}+\kappa_{2} U_{2}+\kappa_{3} E_{z}
$$

where $\kappa_{1}, \kappa_{2}, \kappa_{3} \in \mathbb{R}$. It follows that

$$
D=\kappa_{3}\left|\begin{array}{ccc}
x_{1} & y_{1} & z_{1} \\
x_{2} & y_{2} & z_{2} \\
0 & 0 & 1
\end{array}\right|
$$


Hence, if the sign of $\kappa_{3}$ is known, the problem is reduced to evaluating the sign of

$$
\left|\begin{array}{ccc}
x_{1} & y_{1} & z_{1} \\
x_{2} & y_{2} & z_{2} \\
0 & 0 & 1
\end{array}\right|=\left|\begin{array}{ll}
x_{1} & y_{1} \\
x_{2} & y_{2}
\end{array}\right|,
$$

and we are faced with a 2-dimensional problem.

We now show that, in some (usually most) cases, the sign of $\kappa_{3}$ can be determined. We have:

$$
\kappa_{3}=z_{3}-\kappa_{1} z_{1}-\kappa_{2} z_{2} .
$$

Let $\kappa_{1}=k_{1}+\rho_{1}, \kappa_{2}=k_{2}+\rho_{2}$, with $k_{1}=\left\lfloor\kappa_{1}\right\rfloor, k_{2}=\left\lfloor\kappa_{2}\right\rfloor$ and $0 \leq \rho_{1}, \rho_{2}<1$. We define

$$
R=U_{3}-k_{1} U_{1}-k_{2} U_{2} .
$$

Then, Equation 3 becomes

$$
\kappa_{3}=z_{R}-\rho_{1} z_{1}-\rho_{2} z_{2} .
$$

If $z_{R}<0$, then $\kappa_{3}<0$ since $z_{1}$ and $z_{2}$ are nonnegative, and, if $z_{R}>z_{1}+z_{2}, \kappa_{3}>0$. Otherwise, $R$ lies in the intersection $\mathcal{B}$ of the cylinder projecting along the $z$ axis onto the parallelogram $u_{1} \oplus u_{2}$ ( $\oplus$ denoting the Minkowski sum) with the slab of points $W$ such that $0 \leq z_{W} \leq z_{1}+z_{2}$ (see Figure 1). In this case, we still do not know the sign of $\kappa_{3}$, but we can write:

$$
D=\left|\begin{array}{ccc}
x_{1} & y_{1} & z_{1} \\
x_{2} & y_{2} & z_{2} \\
x_{R} & y_{R} & z_{R}
\end{array}\right|
$$

where the row with largest $z$ component has been replaced by $R$.

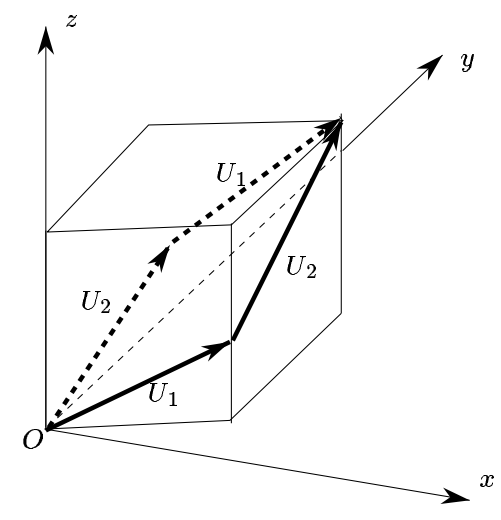

Figure 1: Box $\mathcal{B}$.

The following geometric interpretation of our definitions will be useful in the sequel. Let $H$ be the plane passing through $O$ and spanned 
by $U_{1}$ and $U_{2}$. The vectors $U_{1}$ and $U_{2}$ generate in $H$ the lattice $\mathcal{L}_{H}=$ $\left\{l_{1} U_{1}+l_{2} U_{2}, l_{1}, l_{2} \in \mathbb{Z}\right\} . \quad \mathcal{L}_{H}$ projects vertically onto the lattice $\mathcal{L}$ of the horizontal plane generated by $u_{1}$ and $u_{2}$. To each cell $\mathcal{C}=$ $\left\{\left(l_{1}+\varepsilon_{1}\right) u_{1}+\left(l_{2}+\varepsilon_{2}\right) u_{2}, 0 \leq \varepsilon_{1}, \varepsilon_{2} \leq 1\right\}$ of $\mathcal{L}$, we associate its reference point $l_{1} u_{1}+l_{2} u_{2}$. In particular, $k_{1} u_{1}+k_{2} u_{2}$ is the reference point of the cell of $\mathcal{L}$ that contains $u_{3}$. To each cell of $\mathcal{L}$, we associate also a box. The box associated to the cell $\mathcal{C}$ of $\mathcal{L}$ with reference point $l_{1} u_{1}+l_{2} u_{2}$ is a copy of $\mathcal{B}$ translated by vector $l_{1} U_{1}+l_{2} U_{2}$ which projects vertically onto $\mathcal{C}$. Let $\mathbb{B}$ be the union of these boxes, i.e. the translated copies of $\mathcal{B}$ by the vectors $l_{1} U_{1}+l_{2} U_{2}$ for $l_{1}, l_{2} \in \mathbb{Z}$. $\mathbb{B}$ contains plane $H$ and can be considered as an approximation of $H$.

The geometric interpretation of the above dicussion is now: if $U_{3}$ lies above (below) $\mathbb{B}, \kappa_{3}$ is positive (negative), and otherwise, $U_{3}$ can be replaced by a vector contained in $\mathcal{B}$.

\subsection{The algorithm}

The algorithm consists of $O(b)$ iterations, but may terminate earlier. Each iteration consists of three steps.

During a preliminary step, described in Subsection 3.3, the vectors whose $z$ components are negative are replaced by the opposite vectors and some easy cases are solved.

The first step, described in Subsection 3.4, determines whether $U_{3}$ lies below $\mathbb{B}$, above $\mathbb{B}$, or inside some box of $\mathbb{B}$. In the first two cases, $D$ reduces to a $2 \times 2$ determinant with $b$-bit integer entries : its sign can be evaluated using the algorithm of Section 2 . In the last case, we translate point $U_{3}$ in a direction parallel to $H$ to obtain $R=U_{3}-k_{1} U_{1}-k_{2} U_{2} \in \mathcal{B}$. However, we cannot simply iterate on the vectors $\left(U_{1}, U_{2}, R\right)$. Indeed, although $R$ is known to lie in box $\mathcal{B}$, the binary representation of its components may require as many as $b+1$ bits. Furthermore, the $z$ component $z_{R}$ of $R$ only satisfies $0 \leq z_{R} \leq z_{1}+z_{2}$, which does not imply that this component is smaller than the original $z_{3}$.

The second step of the algorithm, to be described in Subsection 3.6, will either evaluate the sign of $\kappa_{3}$ or find a vector $R^{\prime}=R+\theta_{1} U_{1}+\theta_{2} U_{2}$ $\left(\theta_{1}, \theta_{2} \in\{-1,0,+1\}\right)$ such that the encoding length of its $x$ and $y$ components does not exceed $b$, and its $z$ component is less than $z_{3} / 2$.

The algorithm is then iterated on the vectors $\left(U_{1}, U_{2}, R^{\prime}\right)$. Now, a reduction by two of the modulus of the maximum $z$ component of the vectors in $D$ is guaranteed after at most three iterations. Hence, in total, at most $3 b$ iterations will be required to either find that $D=0$ or end up with a $2 \times 2$ determinant.

\subsection{Preliminary step}

As in the two-dimensional case, the sign of the determinant can be evaluated readily in some cases. First, we multiply by -1 the rows whose $z$ component are negative so that all the entries of the last column are non-negative. This does not change $D$ if no or two rows are concerned and changes $D$ to $-D$ otherwise. Secondly, we permute 
the rows so that $U_{3}$ has the largest $z$ component. Again the change in the sign of $D$ induced by the permutation is known.

Then, if the three minors $\left|\begin{array}{ll}x_{1} & y_{1} \\ x_{2} & y_{2}\end{array}\right|,\left|\begin{array}{ll}x_{2} & y_{2} \\ x_{3} & y_{3}\end{array}\right|$ and $\left|\begin{array}{ll}x_{3} & y_{3} \\ x_{1} & y_{1}\end{array}\right|$ are all strictly positive (negative) then $D$ is positive (negative). Geometrically, this case corresponds to the situation where $u_{1}, u_{2}$ and $u_{3}$ span positively the horizontal plane, or, equivalently, the origin lies inside the triangle $u_{1} u_{2} u_{3}$.

\subsection{First step: computing $R$}

The first step of each iteration either determines the sign of $\kappa_{3}$ or, when $U_{3}$ lies in $\mathbb{B}$, computes the vector $R=U_{3}-k_{1} U_{1}-k_{2} U_{2}$. This is not an obvious task since the moduli of the integers $k_{1}$ and $k_{2}$ can be as large as $2^{2 b+1}$ : indeed, $k_{1}=\left\lfloor\kappa_{1}\right\rfloor$ and $k_{2}=\left\lfloor\kappa_{2}\right\rfloor$ and it follows from (1) that

$$
\kappa_{1}=\frac{\left|\begin{array}{ll}
x_{3} & y_{3} \\
x_{2} & y_{2}
\end{array}\right|}{\left|\begin{array}{ll}
x_{1} & y_{1} \\
x_{2} & y_{2}
\end{array}\right|} \text { and } \kappa_{2}=\frac{\left|\begin{array}{ll}
x_{1} & y_{1} \\
x_{3} & y_{3}
\end{array}\right|}{\left|\begin{array}{ll}
x_{1} & y_{1} \\
x_{2} & y_{2}
\end{array}\right|}
$$

As we will restrict ourselves to $(b+1)$-bit arithmetic (a full discussion of that point will be given in Section 3.5), we may not be able to compute the $k_{i}$.

Consider again the lattice $\mathcal{L}$ in the horizontal plane generated by $u_{1}$ and $u_{2}$. As vector $U_{3}$ has $b$-bit integer components, the coordinates of the points of the line segments $\mathrm{OU}_{3}$ and $\mathrm{Ou}_{3}$ have moduli less than $2^{b}$.

Informally, our strategy to compute $R$ is to probe a subset of $O(b)$ edges of the lattice $\mathcal{L}$ crossed by $O u_{3}$. For each such edge, we consider the corresponding edge in lattice $\mathcal{L}_{H}$. If the $z$ range of all edges encountered during the process remain inside the bounds of the available $(b+1)$-bit arithmetic, the procedure ends when the cell containing $u_{3}$ is found. If, on the contrary, an encountered edge of $\mathcal{L}_{H}$ belongs to a box whose $z$ range exceeds the $(b+1)$-bit representation, the $z$ range of this edge extends entirely outside the bounds of the $b$-bit representation. In such a case, the sign of $\kappa_{3}$ can be determined easily and the calculation of $R$ is halted.

We now present the details of the procedure.

Substep 1.1. Consider the parallelogram formed by the union of the four lattice cells $\mathcal{C}_{0}, \mathcal{C}_{0}-u_{1}, \mathcal{C}_{0}-u_{2}, \mathcal{C}_{0}-\left(u_{1}+u_{2}\right)$, where $\mathcal{C}_{0}$ is the Minkowski sum $u_{1} \oplus u_{2}$. The boundary of this parallelogram consists of eight cell edges. By a straightforward (three-step) binary search we determine which of these edges is intersected by the half-line $L$ issued from $O$ and containing $u_{3}$, the search discriminant being the sign of a $2 \times 2$ determinant of the form $\left|\begin{array}{c}u_{3} \\ w\end{array}\right|$ where $w \in\left\{u_{1}, u_{2}, u_{1}+u_{2}, u_{1}-\right.$ $\left.u_{2}\right\}$. This search also identifies which of the cells $\mathcal{C}_{0}, \mathcal{C}_{0}-u_{1}, \mathcal{C}_{0}-u_{2}$, $\mathcal{C}_{0}-\left(u_{1}+u_{2}\right)$ is intersected by the half-line $L$. Let $\mathcal{C}_{0}^{\prime}=\mathcal{C}_{0}-\epsilon_{1} u_{1}-\epsilon_{2} u_{2}$ 
be such cell. Note that if any of the determinants $\left|\begin{array}{c}u_{3} \\ w\end{array}\right|$ is equal to 0 , then either $k_{1}= \pm k_{2}$ or one of the $k_{i}$ is 0 . In such cases, the sign of $\kappa_{3}$ or $R$ can be computed as in the two-dimensional case.

For the sake of simplicity, we standardize the problem by replacing the original basis $\left(u_{1}, u_{2}\right)$ with the basis $\left(v_{1}, v_{2}\right)$, where $v_{i}=\left(-2 \epsilon_{i}+\right.$ 1) $u_{i}, i=1,2$. Denoting by $c_{u}$ (resp. $c_{v}$ ) the reference point of the cell $\mathcal{C}$ of $\mathcal{L}$ that contains $u_{3}$ when we take $\left(u_{1}, u_{2}\right)$ (resp. $\left(v_{1}, v_{2}\right)$ ) as basis vectors of $\mathcal{L}$, we observe that

$$
c_{u}=c_{v}+\epsilon_{1} u_{1}+\epsilon_{2} u_{2} .
$$

We shall in fact compute $c_{v}$, and then obtain $c_{u}$ using (5). In the sequel, $k_{1}^{\prime}$ and $k_{2}^{\prime}$ will denote the coordinates of $c_{v}$ in the basis $\left(v_{1}, v_{2}\right)$. Let $D_{i}, i=1,2$, be the line of the horizontal plane containing $v_{i}$.

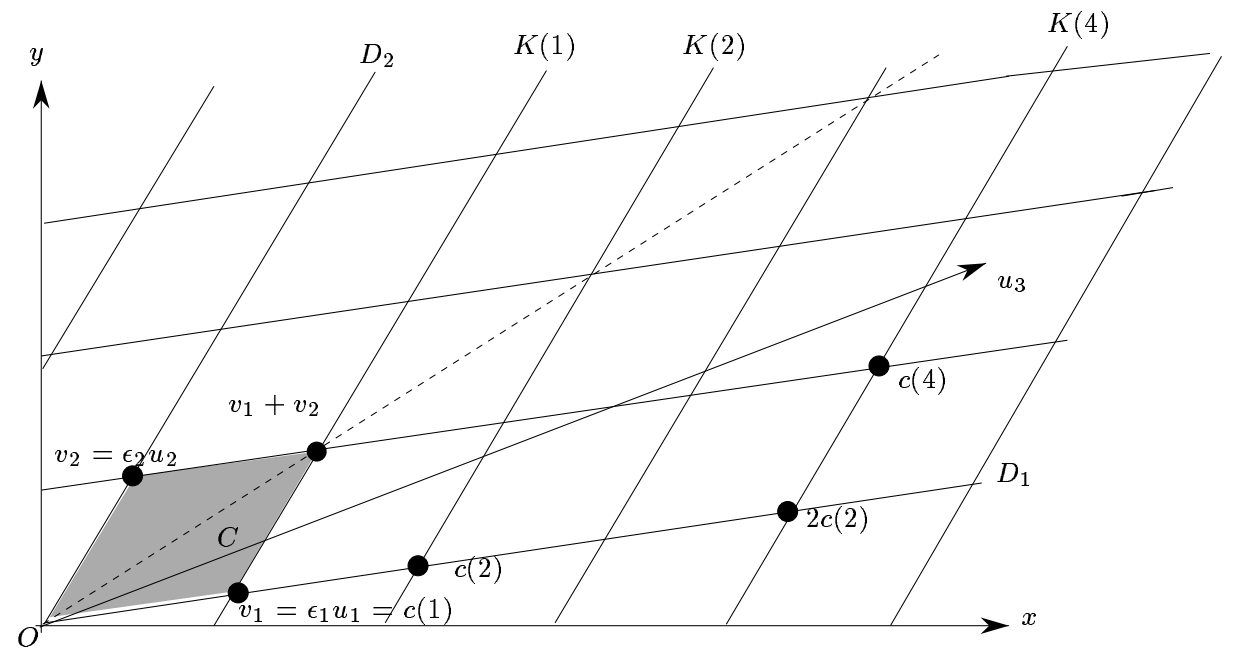

Figure 2: Illustration of Step 1.

Substep 1.2. Next, we have to test whether or not $u_{3}$ belongs to cell $\mathcal{C}_{0}^{\prime}$. This test entails locating $u_{3}$ with respect to the edge traversed by the half-line $L$, i.e. locating $u_{3}$ with respect to either $D_{1}+v_{2}$ or $D_{2}+v_{1}$. In the first case, we evaluate the sign of $\left|\begin{array}{c}v_{1} \\ u_{3}-v_{2}\end{array}\right|$ and, in the second, of $\left|\begin{array}{c}u_{3}-v_{1} \\ v_{2}\end{array}\right|$ (if the sign is negative, $u_{3} \in \mathcal{C}_{0}^{\prime}$ ). If $u_{3}$ is included in $\mathcal{C}_{0}^{\prime}, k_{1}^{\prime}=k_{2}^{\prime}=0$, we deduce $c_{u}$ and the corresponding point $C_{u}$ of $\mathcal{L}_{H}$ using (5), compute $R=U_{3}-C$ and go to Substep 1.6. Otherwise, we proceed to Substep 1.3.

Substep 1.3. Let $K(1)$ denote the first lattice line traversed by $O u_{3}$, i.e., $K(1) \in\left\{D_{1}+v_{2}, D_{2}+v_{1}\right\}$. Without loss of generality, here 
and hereafter, we assume $K(1)=D_{2}+v_{1}$ (see figure 2). For $\lambda \in \mathbb{N}$, we note $K(\lambda)$ the line $D_{2}+\lambda v_{1}$. The vertices of the edge of lattice $\mathcal{L}$ belonging to $K(\lambda)$ and intersected by $O u_{3}$ (if such an intersection exists) are denoted $c(\lambda)$ and $c(\lambda)+v_{2}$. Notice that $c(\lambda)=\lambda v_{1}+\lambda^{\prime} v_{2}$ for some $\lambda^{\prime} \in \mathbb{N}, 0 \leq \lambda^{\prime}<\lambda$.

Notice that like $k_{1}$ and $k_{2}, \lambda$ and $\lambda^{\prime}$ may be as large as $2^{2 b+1}$ but since they would not be explicitly computed this is not a problem.

In this substep, we successively probes lines $K(2), K(4) \ldots K\left(2^{i}\right) \ldots$ Each of these probes may yield the sign of $\kappa_{3}$, in which case the process terminates. If not, the search continues until the unique integer $k$ is determined such that $O u_{3}$ intersects $K\left(2^{k}\right)$ but not $K\left(2^{k+1}\right)$. After completing the probe of $K\left(2^{j}\right)$, the algorithm stores in a stack $S$ point $c\left(2^{j}\right)$. It is convenient to describe the search as a sequence of simpler actions detailed below.

1.3.1. Assume that $c\left(2^{l-1}\right)$ has been determined. $O u_{3}$ crosses the line $K\left(2^{l-1}\right)$ between $c\left(2^{l-1}\right)$ and $c\left(2^{l-1}\right)+v_{2}$. Let $z^{\prime}$ and $z^{\prime \prime}$ be the $z$ coordinates of the two corresponding points of the lattice $\mathcal{L}_{H}$, $\left|z^{\prime}-z^{\prime \prime}\right|=z_{2}$. If $z^{\prime}$ and $z^{\prime \prime}$ are both negative, then $\kappa_{3}>0$ and, if $z^{\prime}$ and $z^{\prime \prime}$ are both greater than $2^{b}$, then $\kappa_{3}<0$; in such cases, the sign of $D$ is known and the algorithm halts. Otherwise $z^{\prime}$ and $z^{\prime \prime}$ have both encoding lengths at most $b+1$, and the process continues.

1.3.2. We test if $O u_{3}$ crosses the line $K\left(2^{l}\right)$ by evaluating the sign of the $2 \times 2$ determinant $\Delta=\left|\begin{array}{c}u_{3}-2 c\left(2^{l-1}\right) \\ v_{2}\end{array}\right|$. Notice that $2 c\left(2^{l-1}\right) \in K\left(2^{l}\right)$. If $O u_{3}$ does not intersect $K\left(2^{l}\right)(\Delta<0)$, then we go to Substep 1.4. Otherwise, we compute $c\left(2^{l}\right)$ as described in Substep 1.3.3.

1.3.3. Since $O u_{3}$ crosses $K\left(2^{l-1}\right)$ between $c\left(2^{l-1}\right)$ and $c\left(2^{l-1}\right)+v_{2}$, $O u_{3}$ crosses $K\left(2^{l}\right)$ between $2 c\left(2^{l-1}\right)$ and $2 c\left(2^{l-1}\right)+2 v_{2}$. Clearly $c\left(2^{l}\right)$ is either $2 c\left(2^{l-1}\right)$ or $2 c\left(2^{l-1}\right)+v_{2}$ (the latter in Figure 3$)$. Point $c\left(2^{l}\right)$ can be determined by testing on which side of $O u_{3}$ point $2 c\left(2^{l-1}\right)+v_{2}$ lies, i.e., by evaluating the sign of the $2 \times 2$ determinant $\left|\begin{array}{c}u_{3} \\ 2 c\left(2^{l-1}\right)+v_{2}\end{array}\right|$. Go to 1.3.1

Substep 1.4. Assume now that $O u_{3}$ intersects the line $K\left(2^{k}\right)$ but not the line $K\left(2^{k+1}\right)$. Then the algorithm computes the integer $\lambda_{f}$ such that $O u_{3}$ crosses $K\left(\lambda_{f}\right)$ but not $K\left(\lambda_{f}+1\right)$. The determination of $\lambda_{f}$ is a binary search. This search involves $k$ steps numbered $k-1, k-2, \ldots, 0$. We denote $\lambda_{h}$ the integer such that $O u_{3}$ intersects $K\left(\lambda_{h}\right)$ but not $K\left(\lambda_{h}+2^{h}\right)$. Assume that at the beginning of step $h$, we know $\lambda_{h+1}$ and $c\left(\lambda_{h+1}\right)$. We now explain how $\lambda_{h}$ and $c\left(\lambda_{h}\right)$ are inductively computed from $\lambda_{h+1}$ and $c\left(\lambda_{h+1}\right)$. The basis of the induction is given by $h+1=k$ and $\lambda_{h+1}=2^{k}$. Again, the search is better illustrated as a sequence of simpler actions.

1.4.1 First, as in Step 1.3.1, let $z^{\prime}$ and $z^{\prime \prime}$ be the $z$ coordinates of the two points of the lattice $\mathcal{L}_{H}$ corresponding to $c\left(\lambda_{h+1}\right)$ and $c\left(\lambda_{h+1}\right)+v_{2}$. If $z^{\prime}$ and $z^{\prime \prime}$ are both negative, then $\kappa_{3}>0$ and, if 


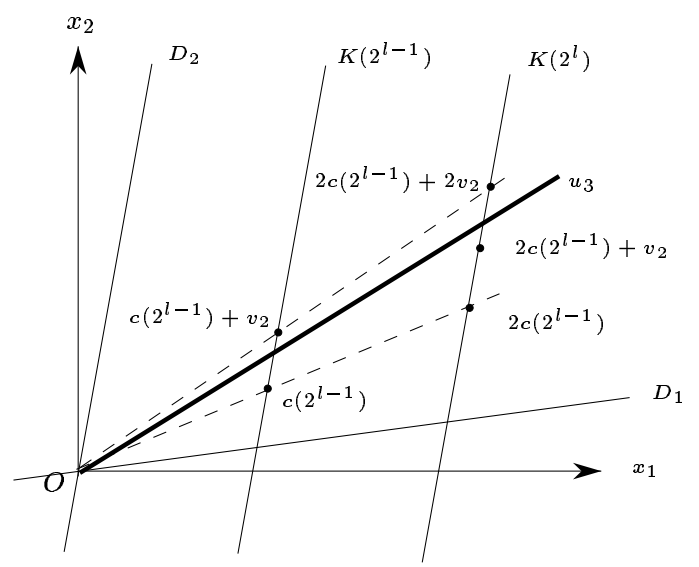

Figure 3: For Substep 1.3.3.

$z^{\prime}$ and $z^{\prime \prime}$ are both greater than $2^{b}$, then $\kappa_{3}<0$; in such cases, the sign of $D$ is known and the algorithm halts. Otherwise $z^{\prime}$ and $z^{\prime \prime}$ have both encoding lengths at most $b+1$.

1.4.2 The algorithm then determines on which side of $K\left(\lambda_{h+1}+2^{h}\right)$ point $u_{3}$ lies, which can be done by evaluating the sign of the $2 \times 2$ determinant $\Delta^{\prime}=\left|\begin{array}{c}u_{3}-c\left(\lambda_{h+1}\right)-c\left(2^{h}\right) \\ v_{2}\end{array}\right|$, where $c\left(2^{h}\right)$ is popped out of stack $S$. If $u_{3}$ lies on the same side of $K\left(\lambda_{h+1}+2^{h}\right)$ as $O\left(\Delta^{\prime}<0\right)$, then $\lambda_{h+1}=\lambda_{h}, c\left(\lambda_{h}\right)=c\left(\lambda_{h+1}\right)$ and we can proceed to Step $h-1$. Otherwise, $\lambda_{h}=\lambda_{h+1}+2^{h}$ and we have to compute $c\left(\lambda_{h}\right)$.

1.4.3 It is to be observed that $O u_{3}$ crosses $K\left(\lambda_{h}\right)$ between $c\left(\lambda_{h+1}\right)+$ $c\left(2^{h}\right)$ and $c\left(\lambda_{h+1}\right)+c\left(2^{h}\right)+2 v_{2}$. Thus $c\left(\lambda_{h}\right)$ is either $c\left(\lambda_{h+1}\right)+$ $c\left(2^{h}\right)$ or $c\left(\lambda_{h+1}\right)+c\left(2^{h}\right)+v_{2}$ depending on which side of $O u_{3}$ point $c\left(\lambda_{h+1}\right)+c\left(2^{h}\right)+v_{2}$ lies, which is given by the sign of $\left|\begin{array}{c}u_{3} \\ c\left(\lambda_{h+1}\right)+c\left(2^{h}\right)+v_{2}\end{array}\right|$. Go to 1.4.1.

The search is carried on until one of the following occurs : the algorithm halts, or it goes to Substep 1.5 at an intermediate stage, or it performs all the steps $k-1, k-2, \ldots, 0$. In the last case, we set $\lambda_{f}=\lambda_{0}$ and go to Substep 1.5.

Substep 1.5 This substep determines the cell of $\mathcal{L}$ that contains $u_{3}$. We know that $u_{3}$ belongs to the parallelogram $c\left(\lambda_{f}\right), c\left(\lambda_{f}\right)+$ $v_{1}, c\left(\lambda_{f}\right)+v_{1}+2 v_{2}, c\left(\lambda_{f}\right)+2 v_{2}$. A last test locates $u_{3}$ with respect to line $D_{1}+c\left(\lambda_{f}\right)+v_{2}$ and determines the cell $\mathcal{C}$ of the lattice $\mathcal{L}$ that contains $u_{3}$. More precisely, if $\left|\begin{array}{c}u_{3}-c\left(\lambda_{f}\right)-v_{2} \\ v_{1}\end{array}\right| \geq 0$, then $\mathcal{C}$ is the cell whose reference point is $c\left(\lambda_{f}\right)$ in the $\left(v_{1}, v_{2}\right)$ basis. Otherwise, $\mathcal{C}$ is the cell whose reference point is $c\left(\lambda_{f}\right)+v_{2}$. The reference point $c_{u}$ of $\mathcal{C}$ in the $\left(u_{1}, u_{2}\right)$ basis and the corresponding point $C_{u}$ of $\mathcal{L}_{H}$ are then obtained using (5) and we compute $R=U_{3}-C_{u}$. 
Substep 1.6 If $z_{R}<0$, then $\kappa_{3}<0$ and, if $z_{R}>z_{1}+z_{2}$, then $\kappa_{3}>0$. Otherwise, we go to Step 2 (to be described in Section 3.6).

This ends the description of the first major step of the algorithm. The vector $r=u_{3}-c_{u}$ belongs to the cell of $\mathcal{L}$ whose reference point is $O$, thus the encoding lengths of $x_{R}$ and $y_{R}$ are at most $b+1$ and $0 \leq z_{R} \leq z_{1}+z_{2}$.

\subsection{Arithmetic}

In this section, we show that $(b+1)$-bit arithmetic is sufficient to run the above algorithm, assuming that $U_{1}, U_{2}$ and $U_{3}$ are $b$-bit integers.

First, we observe that all encountered $c(\lambda)$ and $c(\lambda)+v_{2}$ can be represented using $b+1$ bits. Indeed, since $p=K(\lambda) \cap O u_{3}$ belongs to the line segment $O u_{3}$, the encoding length of its coordinates is less than the one of $u_{3}$, and since $c(\lambda)$ and $c(\lambda)+v_{2}$ belong to the line segment $p-u_{2}, p+u_{2}$, one additional bit is enough to store $c(\lambda)$ and $c(\lambda)+v_{2}$. In the case where $c(\lambda)$ is computed as the sum of two terms $c\left(\lambda_{1}\right)+c\left(\lambda_{2}\right)$, then clearly $c(\lambda)$ can be computed without difficulty. In the other case where $c(\lambda)=\left[c\left(\lambda_{1}\right)+c\left(\lambda_{2}\right)\right]+v_{2}(b+2)$ bits may be required to store the intermediate result $c\left(\lambda_{1}\right)+c\left(\lambda_{2}\right)$, but fortunately, among the three possibilities $c(\lambda)=\left[c\left(\lambda_{1}\right)+c\left(\lambda_{2}\right)\right]+v_{2}, c(\lambda)=c\left(\lambda_{1}\right)+\left[c\left(\lambda_{2}\right)+v_{2}\right]$ and $c(\lambda)=c\left(\lambda_{2}\right)+\left[c\left(\lambda_{1}\right)+v_{2}\right]$ there always exists one that allows to compute the $x$ (resp. $y, z$ ) coordinate of the sum so that the intermediate result remains on $b+1$ bits (the formulas may be different for the different coordinates).

Next, we show that the computations of vector $R$ performed in Substeps 1.2 or 1.5 do not require more than $(b+1)$-bit arithmetic. The computation of the $x$ and $y$-components of $R$ do not cause any problem since $c_{v}-c_{u}, u_{3}-c_{v}$ and $u_{3}-c_{u}$ can each be represented with at most $b+1$ bits. Consider now the computation of $z_{R}$. Let $z_{v}$ be the $z$-component of the point $C_{v}$ of $\mathcal{L}_{H}$ which corresponds to $c_{v}$ and let $z_{u}$ be the $z$-component of $C_{u}$. If $z_{R}=z_{3}-z_{u}=z_{3}-z_{v}-\varepsilon_{1} z_{1}-\varepsilon_{2} z_{2}$ is in the range $\left[-2^{b+1}, 2^{b+1}\right]$; then can be computed, (indeed there is a way to organize the above sum in order to compute it using $(b+1)$-bit arithmetic); else $\left(\left|z_{R}\right|>2^{b+1}\right)$, we can easily decide if $z_{R}$ is positive or negative $\left(\kappa_{3}\right.$ has the same sign as $\left.z_{R}\right)$.

The bit-length of $z_{v}$ is known to be at most $b+1$, and it is easy to see from Equation (5) that the bit-length of $z_{u}$ does not exceed the bit-length of $z_{v}$. If $z_{u}$ is positive or negative with $\left|z_{u}\right|<2^{b}, z_{R}=z_{3}-z_{u}$ can be computed using $(b+1)$-bit arithmetic. If $z_{u}$ is negative with $\left|z_{u}\right|>2^{b}$, then $z_{R}$ and $\kappa_{3}$ are known to be negative and we do not need to compute $z_{R}$.

At last, we show that the signs of the determinants used in Steps 1.3.2, 1.3.3, 1.4.2 and 1.4.3 can be evaluated using $(b+1)$-bit arithmetic. The vectors appearing in those determinants are combinations of $u_{1}$, $u_{2}, u_{3}$ and some $c(\lambda)$ : for instance, at Step 1.3.3, we need to compute $2 c\left(2^{l-1}\right)+v_{2}$. It follows from the description of the algorithm that these vectors can always be computed using $(b+3)$-bit arithmetic. We now explain how to reduce the number of bits of the arithmetic to $b+1$ 
for each of the Steps 1.3.2, 1.3.3, 1.4.2 and 1.4.3 of the algorithm.

Step 1.3.2. In this Step, we test on which side of $K\left(2^{l}\right)$ point $u_{3}$ lies. The problem arises when the coordinates of $u_{3}-2 c\left(2^{l-1}\right)$ are not both representable with $b+1$ bits. The idea is therefore to replace point $2 c\left(2^{l-1}\right)$ with another point $w$ of $K\left(2^{l}\right)$ such that $u_{3}-w$ is represented with $b+1$ bits. Let $\mathcal{S P} \triangleq\left[-2^{b}, 2^{b}\right] \times\left[-2^{b}, 2^{b}\right]$ be the single precision domain. We claim that such a construction can be accomplished if at least one of $\left\{c\left(2^{l-1}\right), c\left(2^{l-1}\right)+v_{2}\right\}$ and at least one of $\left\{c\left(2^{l-1}\right)-u_{3}, c\left(2^{l-1}\right)+v_{2}-u_{3}\right\}$ belong to $\mathcal{S P}$. Let $w_{1}$ and $w_{2}-u_{3}$ be points in $\mathcal{S P}$ choosen from these two sets respectively. It follows that point $-w_{1}-\left(w_{2}-u_{3}\right)=u_{3}-\left(w_{1}+w_{2}\right)$ is representable with $b+1$ bits and that $w_{1}+w_{2} \in K\left(2^{l}\right)$ since $w_{1}, w_{2} \in K\left(2^{l-1}\right)$. We conclude that the entries of the determinant $\left|\begin{array}{c}u_{3}-w \\ v_{2}\end{array}\right|$ are representable with $b+1$ bits. Otherwise we have the following easily decidable alternatives :

(i) $c\left(2^{l-1}\right) \notin \mathcal{S P}$ et $c\left(2^{l-1}\right)+v_{2} \notin \mathcal{S P}$. We claim that $u_{3}$ and $O$ are on the same side of $K\left(2^{l}\right)$. Indeed, since $v_{2} \in \mathcal{S P}$, the line $K\left(2^{l-1}\right)$ does not intersect the square $\mathcal{S} \mathcal{P}^{*}$ with vertices $\left(0, \pm 2^{b}\right)$ and $\left( \pm 2^{b}, 0\right)$ (see Figure $4 a$ ). Then $K\left(2^{l}\right)$ is entirely outside the square $2 \mathcal{S P}^{*}$ which contains $\mathcal{S P}$.

(ii) $c\left(2^{l-1}\right)-u_{3} \notin \mathcal{S P}$ and $c\left(2^{l-1}\right)+v_{2}-u_{3} \notin \mathcal{S P}$. We claim that $u_{3}$ and $O$ are on distincts sides of $K\left(2^{l}\right)$. Indeed, arguing as above, line $K\left(2^{l-1}\right)$ does not intersect the square $\mathcal{S P}^{*}+u_{3}\left(\mathcal{S P}^{*}\right.$ centered at $u_{3}$, see Figure $\left.4 \mathrm{~b}\right)$. Thus, if $p$ is the intersection between $K\left(2^{l-1}\right)$ and $O u_{3},\left\|p u_{3}\right\|_{\infty} \geq \frac{1}{2}\left\|p u_{3}\right\|_{1}>2^{b-1}$ (since $p$, being external to $\mathcal{S P}^{*}+u_{3}$, has $L_{1}$-distance from $u_{3}>2^{b}$ ). The point $p^{\prime}=2 p$ is the point where the lines $O u_{3}$ and $K\left(2^{l}\right)$ intersect and we have :

$$
\begin{aligned}
\left\|O p^{\prime}\right\|_{\infty} & =2\|O p\|_{\infty}=2\left\|O u_{3}\right\|_{\infty}-2\left\|p u_{3}\right\|_{\infty} \\
& <2\left\|O u_{3}\right\|_{\infty}-2^{b} \leq\left\|O u_{3}\right\|_{\infty},
\end{aligned}
$$

which proves the claim.

Step 1.4.2. The modification of this step is similar to (and simpler than) that of Step 1.3.2. Here we are led to the evaluation of the sign of $\left|\begin{array}{c}u_{3}-w \\ v_{2}\end{array}\right|$, where point $w$ must be chosen on line $K\left(\lambda_{h+1}+2^{h}\right)$. Since, as we already know, $u_{3}$ and $O$ are on distinct sides of $K\left(2^{h+1}\right)$, one of $c\left(2^{h}\right)$ and $c\left(2^{h}\right)+v_{2}$ can be represented with $b$ bits ; let $w_{1}$ be this vector. Similary, since $O$ and $u_{3}$ are on the same side of $K\left(2 \lambda_{h+1}\right)$, one of $c\left(\lambda_{h+1}\right)-u_{3}$ and $c\left(\lambda_{h+1}\right)+v_{2}-u_{3}$ can be represented with $b$ bits ; let $w_{2}-u_{3}$ be this vector. The opposite of the sum of these two vectors $-\left(w_{1}+\left(w_{2}-u_{3}\right)\right)=u_{3}-\left(w_{1}+w_{2}\right)$ is represented with $b+1$ bits and $w \triangleq w_{1}+w_{2} \in K\left(\lambda_{h+1}+2^{h}\right)$, as we wished to show.

Steps 1.3.3 and 1.4.3. The entries of the determinant $\left|\begin{array}{c}u_{3} \\ 2 c\left(2^{l-1}\right)+v_{2}\end{array}\right|$ used in Step 1.3 .3 can be represented with $b+1$ bits since $2 c\left(2^{l-1}\right)+v_{2}$ is either $c\left(2^{l}\right)$ or $c\left(2^{l}\right)+v_{2}$. And the same holds for the entries of the determinant used at Step 1.4.3. 
(a)

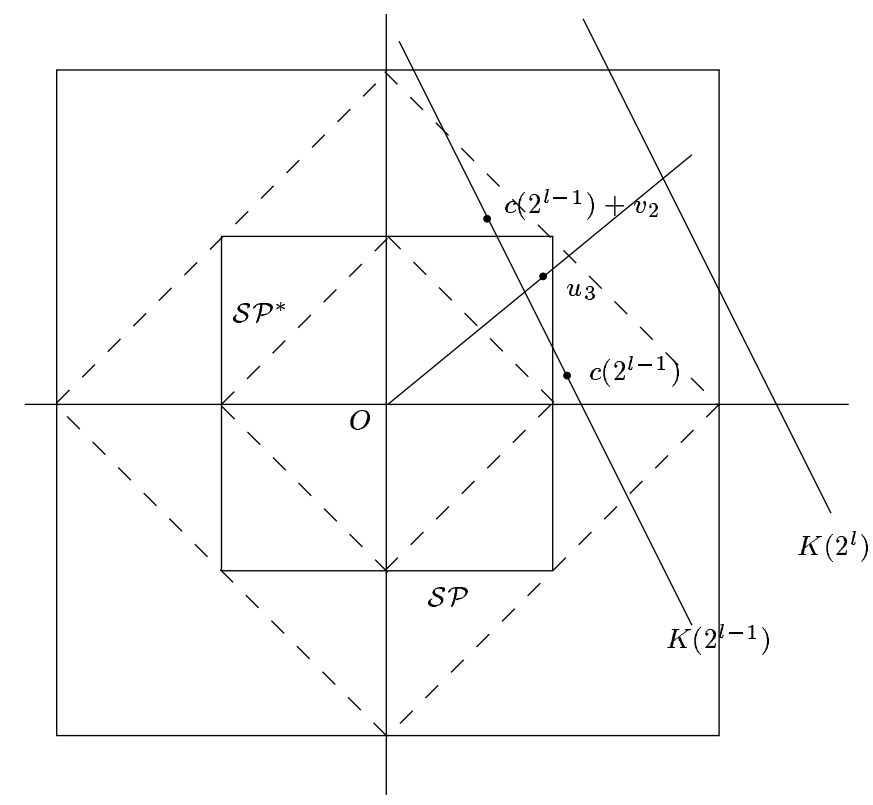

(b)

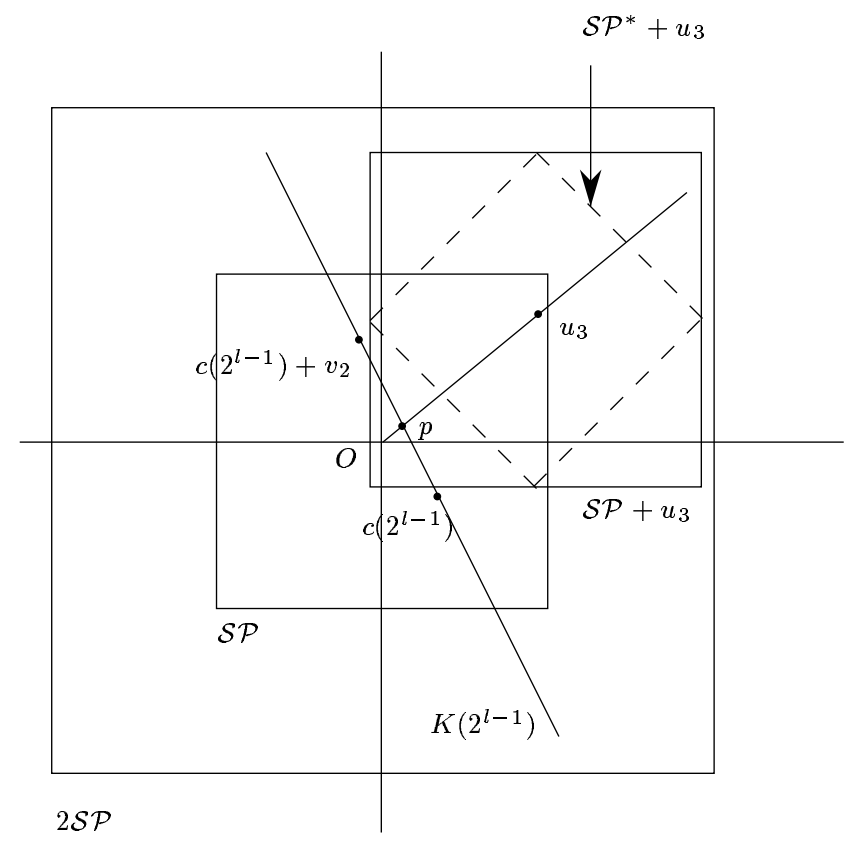

Figure 4: Illustrations for the cases : (a) $c\left(2^{l-1}\right) \notin \mathcal{S P}$ and $c\left(2^{l-1}\right)+v_{2} \notin \mathcal{S P}$, (b) $c\left(2^{l-1}\right)-u_{3} \notin \mathcal{S P}$ and $c\left(2^{l-1}\right)+v_{2}-u_{3} \notin \mathcal{S P}$. 


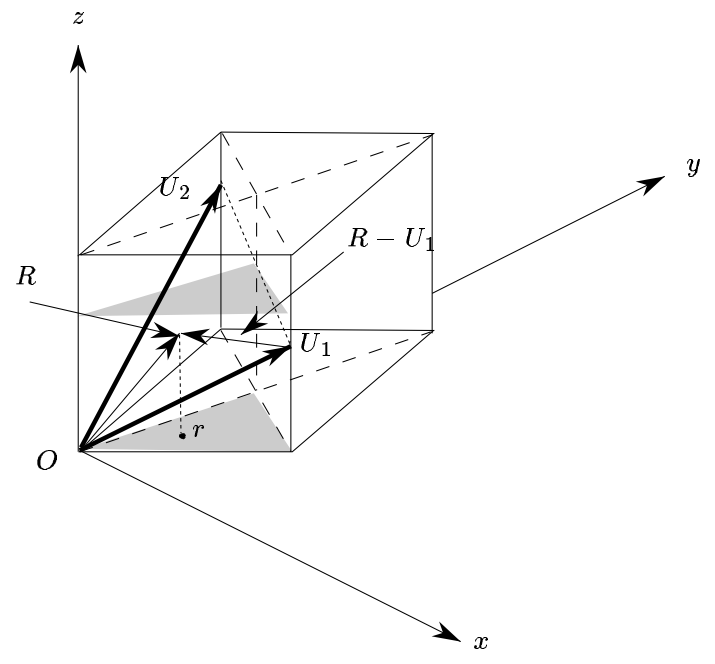

Figure 5: Illustration of the selection of $R^{\prime}$.

\subsection{Second step: exponential reduction}

The second major step of the algorithm, to be described in this subsection, either evaluates the sign of $\kappa_{3}$ or finds a vector $R^{\prime}=R-$ $\theta_{1} U_{1}-\theta_{2} U_{2}, \theta_{1}, \theta_{2} \in\{-1,0,1\}$ such that $x_{R^{\prime}}, y_{R^{\prime}}$ are $b$-bit integers and $\left|z_{R^{\prime}}\right| \leq \frac{z_{3}}{2}$. In order to do that, we will further subdivide the box $\mathcal{B}$ into four sub-boxes such that, for any $r$ lying in each of those sub-boxes, either such a vector $R^{\prime}$ can be determined or the sign of $\kappa_{3}$ can be readily obtained.

The projection of box $\mathcal{B}$ onto the plane is the parallelogram $u_{1} \oplus u_{2}$. The algorithm locates the projection $r$ of $R$ with respect to the two diagonals of the parallelogram joining $u_{1}$ to $u_{2}$ and $O$ to $u_{1}+u_{2}$ by evaluating the sign of $2 \times 2$ determinants $\left|\begin{array}{c}r \\ u_{1}+u_{2}\end{array}\right|$ and $\left|\begin{array}{c}r-u_{1} \\ u_{2}-u_{1}\end{array}\right|$ (see Figure 5).

We illustrate the step just for one of the four sub-boxes; handling of the other three cases is trivially analogous. Assume therefore, without loss of generality, that $r$ belongs to triangle $\left(O, u_{1}, \frac{u_{1}+u_{2}}{2}\right)$ (the shaded triangle in Figure 5). Then

- if $z_{R}>\sup \left(z_{1}, \frac{z_{1}+z_{2}}{2}\right)$, then $\kappa_{3}>0$,

- else, we choose $R^{\prime}$ as the vector among the two vectors $R$ and $R-U_{1}$ whose $z$ component has the smaller modulus. It follows that

$$
\left|z_{R^{\prime}}\right| \leq \frac{\sup \left(z_{1}, z_{2}\right)}{2} \leq \frac{z_{3}}{2} .
$$




\subsection{Nonindependent vectors}

We have assumed above that $\left(u_{1}, u_{2}\right)$ are linearly independent vectors. If these vectors are not independent, the minor $\left|\begin{array}{ll}x_{1} & y_{1} \\ x_{2} & y_{2}\end{array}\right|$ of $D=$ $\left|\begin{array}{lll}x_{1} & y_{1} & z_{1} \\ x_{2} & y_{2} & z_{2} \\ x_{3} & y_{3} & z_{3}\end{array}\right|$ associated to the $z_{3}$ component of $u_{3}$ vanishes and we can simply replace $z_{3}$ by 0 without modifying the determinant:

$$
D=\left|\begin{array}{ccc}
x_{1} & y_{1} & z_{1} \\
x_{2} & y_{2} & z_{2} \\
x_{3} & y_{3} & 0
\end{array}\right|
$$

\subsection{Complexity analysis of the algorithm}

At each iteration, either the algorithm evaluates the sign of $\kappa_{3}$ and ends by the computation of a $2 \times 2$ determinant, or it iterates with a $3 \times 3$ determinant where the greatest (in absolute value) element of the last column has been divided by at least two while the others remain unchanged. It follows that the number of iterations is at most $3 b$.

Each iteration consists of Substeps 1.1-1.6 and Step 2. The cost of each step is dominated by evaluating signs of $2 \times 2$ determinants with $(b+1)$-bit integer entries. Substep 1.1 requires three such evaluations, Substep 1.2 one, Substep 1.3 at most $4 b$ (each iteration requires to evaluate the sign of two determinants and there are at most $\log k_{i} \leq$ $2 b$ iterations), Substep 1.4 at most $4 b$, Substep 1.5 at most one, and Substep 2 at most two. Thanks to Theorem 1, we have the following theorem.

Theorem 2 Let $D$ be $a 3 \times 3$ determinant with $b$-bit integer entries. There exists an algorithm that evaluates the sign of $D$ using only $(b+1)$-bit arithmetic. The algorithm requires at most $3 b$ iterations, each iteration involving the evaluation of at most $8 b+9$ signs of $2 \times 2$ determinants with $(b+1)$-bit integer entries. In the worst-case, the algorithm requires at most $3 b^{2}(8 b+9)$ elementary steps, each elementary step involving $O(1)$ additions/subtractions, comparisons and euclidean divisions.

\section{Geometric applications}

Most geometric tests can be reduced to computing the sign of a determinant. This section shows such reductions for the most basic geometric tests.

\section{1 which_side}

Given $d$ points $A_{1}, \ldots, A_{d}$ in $d$-space, and another point $X$ of $\mathbb{R}^{d}$, Function which_side determines whether $X$ belongs to the hyperplane $H$ passing through the $A_{i}$ or, otherwise, to which half space limited by 
$H$. This function is at the core of many geometric algorithms, and, in particular, of all algorithms computing convex hulls of points in $\mathbb{R}^{d}$.

Function which_side can be implemented as the evaluation of the sign of the $d \times d$ determinant

$$
D=\left|\begin{array}{c}
A_{1}-X \\
\vdots \\
A_{d}-X
\end{array}\right|
$$

with $(b+1)$-bit integer entries, if the points have $b$-bit integer coordinates.

\section{2 sign_dot_product}

Given two 2-vectors $U_{1}=\left(x_{1}, y_{1}\right)$ and $U_{2}=\left(x_{2}, y_{2}\right)$ with $b$-bit integer components, one can determine the sign of the dot product $U_{1} \cdot U_{2}$ by determining the sign of the $2 \times 2$ determinant with $b$-bit integer entries

$$
\left|\begin{array}{cc}
y_{1} & -x_{1} \\
x_{2} & y_{2}
\end{array}\right|=U_{1} \cdot U_{2}
$$

Given two 3-vectors $U_{1}=\left(x_{1}, y_{1}, z_{1}\right)$ and $U_{2}=\left(x_{2}, y_{2}, z_{2}\right)$ with $b$-bit integer components, if $x_{1} \neq 0$, one can determine the sign of the dot product $U_{1} \cdot U_{2}$ by determining the sign of the $3 \times 3$ determinant with $b$-bit integer entries

$$
\left|\begin{array}{ccc}
y_{1} & -x_{1} & 0 \\
z_{1} & 0 & -x_{1} \\
x_{2} & y_{2} & z_{2}
\end{array}\right|=x_{1} U_{1} \cdot U_{2} .
$$

If $x_{1}=0$, the problem is reduced to the 2-dimensional case (this formula can be generalized to higher dimensions).

These results immediately apply for comparing the norms of two vectors. Indeed, given two vectors $U_{1}$ and $U_{2}$,

$$
\left|U_{1}\right|^{2}-\left|U_{2}\right|^{2}=\left(U_{1}+U_{2}\right) \cdot\left(U_{1}-U_{2}\right) .
$$

It follows that comparing the norms of two $d$-vectors with $b$-bit integer components, is equivalent to evaluating the sign of a $d \times d$ determinant with $(b+1)$-bit integer entries.

\section{3 in_circle}

The basic numerical test involved in the construction of Voronoi diagrams in the plane is the following. Given are three points $A_{i}=\left(x_{i}, y_{i}\right)$, $i=1,2,3$, and another point $X=(x, y)$ with $b$-bit integer coordinates. The test consists in deciding whether $X$ lies on the circle $C$ passing through $A_{1}, A_{2}$ and $A_{3}$, inside $C$, or outside $C$. This is equivalent to determining the sign of the following $3 \times 3$ determinant

$$
\text { in_circle }(X)=\left|\begin{array}{ccc}
x_{1}-x & x_{2}-x & x_{3}-x \\
y_{1}-y & y_{2}-y & y_{3}-y \\
x_{1}^{2}+y_{1}^{2}-x^{2}-y^{2} & x_{2}^{2}+y_{2}^{2}-x^{2}-y^{2} & x_{3}^{2}+y_{3}^{2}-x^{2}-y^{2}
\end{array}\right|
$$




$$
\begin{aligned}
& =\left|\begin{array}{ccc}
x_{1}-x & x_{2}-x & x_{3}-x \\
y_{1}-y & y_{2}-y & y_{3}-y \\
0 & \left(x_{2}-x\right)\left(x_{2}-x_{1}\right)+\left(y_{2}-y\right)\left(y_{2}-y_{1}\right) & \left(x_{3}-x\right)\left(x_{3}-x_{1}\right)+\left(y_{3}-y\right)\left(y_{3}-y_{1}\right)
\end{array}\right| \\
& =\frac{1}{x_{1}-x}\left|\begin{array}{ccc}
x_{1}-x & x_{2}-x & x_{3}-x \\
0 & \left(x_{1}-x\right)\left(y_{2}-y\right)-\left(x_{2}-x\right)\left(y_{1}-y\right) & \left(x_{1}-x\right)\left(y_{3}-y\right)-\left(x_{3}-x\right)\left(y_{1}-y\right) \\
0 & \left(x_{2}-x\right)\left(x_{2}-x_{1}\right)+\left(y_{2}-y\right)\left(y_{2}-y_{1}\right) & \left(x_{3}-x\right)\left(x_{3}-x_{1}\right)+\left(y_{3}-y\right)\left(y_{3}-y_{1}\right)
\end{array}\right| \\
& =\left|\begin{array}{cc}
\left(x_{1}-x\right)\left(y_{2}-y\right)-\left(x_{2}-x\right)\left(y_{1}-y\right) & \left(x_{1}-x\right)\left(y_{3}-y\right)-\left(x_{3}-x\right)\left(y_{1}-y\right) \\
\left(x_{2}-x\right)\left(x_{2}-x_{1}\right)+\left(y_{2}-y\right)\left(y_{2}-y_{1}\right) & \left(x_{3}-x\right)\left(x_{3}-x_{1}\right)+\left(y_{3}-y\right)\left(y_{3}-y_{1}\right)
\end{array}\right|
\end{aligned}
$$

It follows that Function in_circle can be implemented as the evaluation of the sign of a $2 \times 2$ determinant with $(2 b+3)$-bit integer entries.

$A$ similar computation shows that the analogous Function in_sphere can be implemented as the evaluation of the sign of the following $3 \times 3$ determinant with $(2 b+4)$-bit integer entries.

$$
\begin{array}{ccc} 
& \text { in_sphere }(X)=\frac{1}{x_{1}-x} \\
& & \\
\left(x_{1}-x\right)\left(y_{2}-y\right)-\left(x_{2}-x\right)\left(y_{1}-y\right) & \left(x_{1}-x\right)\left(y_{3}-y\right)-\left(x_{3}-x\right)\left(y_{1}-y\right) & \left(x_{1}-x\right)\left(y_{4}-y\right)-\left(x_{4}-x\right)\left(y_{1}-y\right) \\
\left(x_{1}-x\right)\left(z_{2}-z\right)-\left(x_{2}-x\right)\left(z_{1}-z\right) & \left(x_{1}-x\right)\left(z_{3}-z\right)-\left(x_{3}-x\right)\left(z_{1}-z\right) & \left(x_{1}-x\right)\left(z_{4}-z\right)-\left(x_{4}-x\right)\left(z_{1}-z\right) \\
\left(c_{2}-x\right)\left(x_{2}-x_{1}\right)+\left(y_{2}-y\right)\left(y_{2}-y_{1}\right)+\left(z_{2}-z_{2}\right)\left(\left(_{2}-z_{1}\right)\right. & \left.\left(x_{3}-x\right)\left(x_{3}-x_{1}\right)+y_{3}-y_{3}\right)\left(y_{3}-y_{1}\right)+\left(x_{3}-z_{3}\right)\left(z_{3}-z_{1}\right) & \left(x_{4}-x\right)\left(x_{4}-x_{1}\right)+\left(y_{4}-y_{2}\right)\left(y_{4}-y_{1}\right)+\left(z_{4}-z\right)\left(z_{4}-z_{1}\right)
\end{array}
$$

\section{4 intersections_sorting}

When constructing arrangements of line segments in the plane and trapezoidal maps (e.g. by a sweep line algorithm), the following crucial numerical test is used. Let $A_{0} A_{1}, A_{2} A_{3}, A_{4} A_{5}$ and $A_{6} A_{7}$ be four line segments. The test consists in deciding if the $x$-coordinate $x_{I}$ of the intersection point $I$ of $A_{0} A_{1}$ and $A_{2} A_{3}$ is smaller or greater than the $x$-coordinate $x_{J}$ of the intersection point $J$ of $A_{4} A_{5}$ and $A_{6} A_{7}$. If the coordinates of points $A_{i}$ are $b$-bit integers, this test reduces to evaluating the sign of a $2 \times 2$ determinant with $(3 b+3)$-bit integer entries.

Indeed, if $A_{i}=\left(x_{i}, y_{i}\right)$ for $i=0, \ldots, 7$,

$$
x_{I}=\frac{A_{I}}{B_{I}}
$$

where

$$
\begin{gathered}
A_{I}=\left|\begin{array}{cc}
x_{1}-x_{0} & x_{0} y_{1}-x_{1} y_{0} \\
x_{3}-x_{2} & x_{2} y_{3}-x_{3} y_{2}
\end{array}\right| \\
B_{I}=\left|\begin{array}{ll}
x_{1}-x_{0} & y_{1}-y_{0} \\
x_{3}-x_{2} & y_{3}-y_{2}
\end{array}\right|
\end{gathered}
$$

and a similar expression can be found for $x_{J}$. Comparing $x_{I}$ and $x_{J}$ reduces to testing the sign of the $2 \times 2$ determinant $\left|\begin{array}{cc}A_{I} & B_{I} \\ A_{J} & B_{J}\end{array}\right|$ where each element $A_{I}$ and $A_{J}$ are $(3 b+3)$-bit integers while $B_{I}$ and $B_{J}$ are $(2 b+3)$-bit integers.

\section{Implementation and experimental results}

Implementation has been done using $\mathrm{C}++$ and the $\mathrm{C}++$ ATT compiler on a Sun SS5-70. Times have been obtained using the clock command. 
The entries are integers stored in variable of type double. This allows us to manipulate exact 53 bit integers, to benefit of the fast floating point arithmetic of the processor and to easily handle overflows. For $3 \times 3$ determinants we do not apply exactly Theorem 2 and use a simplified algorithm that requires a $(b+2)$-bit arithmetic. More precisely, the algorithm does not look for the right order to compute $c(\lambda)=c\left(\lambda_{1}\right)+c\left(\lambda_{2}\right)+v_{2}$ as mentioned in Section 3.5.

The following table sums up the limits on the precision of the entries for the geometric tests of Section 4 using 32-bit, 53-bit and 64-bit arithmetic.

\begin{tabular}{|c|c|c|c|c|c|c|}
\hline arithmetic & function & line side & plane side & in circle & in sphere & intersection \\
\hline \multicolumn{2}{|c|}{ determinant size } & $2 \times 2$ & $3 \times 3$ & $2 \times 2$ & $3 \times 3$ & $2 \times 2$ \\
\hline 32 & entries size & 31 & 29 & 14 & 14 & $\overline{9}$ \\
\hline 53 & entries size & 52 & 50 & 24 & 23 & $\overline{16}$ \\
\hline 64 & entries size & 63 & 61 & 30 & 30 & $\overline{20}$ \\
\hline
\end{tabular}

Implementation is available through WWW at url: http://www .inria.fr:/prisme/personnel/devillers/anglais/determinant.html

The code has been tested on several kinds of determinants and compared with other methods that compute the determinant and subsequently test the sign.

Two variants of our method have been implemented.

Standard: the algorithm is iterated, until the comparison of the entries and computation of sign of minors in the $3 \mathrm{D}$ case guarantees a conclusion. The evaluation of the sign of $2 \times 2$ determinants in the 3D algorithm are computed using the lazy variant.

Lazy: The algorithm is combined with a floating point filter. In the $2 \mathrm{D}$ case, the IEEE standard ensures that the sign of the determinant can be computed exactly when using the floating point arithmetic is exact as long as it is non zero. Thus the exact arithmetic is invoked only in that case. In the 3D case, if the absolute value of the rounded determinant is bigger than $2^{\beta}$ where $\beta=\log \max \left\|x_{i}\right\|+\log \max \left\|y_{i}\right\|+$ $\log \max \left\|z_{i}\right\|-b+5$ we rely on the sign given by the rounded computation (IEEE norm ensure that the direct computation of the determinant $D$ yields an approximate value $\tilde{D}=D\left(1+5 \varepsilon_{\text {machine }}\right)$. This filter is applied each time a new determinant is considered (i.e. each time some $R^{\prime}$ is substituted to $U_{3}$ ).

\section{Direct computation}

This computation is done by first converting the original data from double to different numeric types and then the determinant is computed using the following formula

$$
\left|\begin{array}{ll}
a & b \\
c & d
\end{array}\right|=a d-b c \text { in the } 2 \mathrm{D} \text { case }
$$

$\left|\begin{array}{lll}a & b & c \\ d & e & f \\ g & h & i\end{array}\right|=(a(e i-f h)+b(f g-d i))+c(d h-e g)$ in the 3D case. 
We have to mention that the type conversion between double and the other types used may be expensive and the time can be reduced if these types are used for the original data (this conversion time is included in the tables below).

The direct computation is run with different arithmetic.

Double: The computation done using double arithmetic is fast but but not sake since it is subject to rounding errors.

Quadruple: The computation is done using quadruple precision (long double type). For $2 \times 2$ determinants (but not for $3 \times 3$ ), this ensures exactness of the computation.

LEDA-integer: LEDA provides exact computation on integers of arbitrary length. The result is exact [MN89].

LN: LN Package, by S. Fortune and C. Van Wyk, works as a compiler, it transforms expression in LN language into optimized $\mathrm{C}++$ routine computing the sign of the expression, first through a filter if it is not enough to conclude using optimized exact arithmetic (of fixed length). The result is exact [FV94].

\section{Input}

These methods have been tested and compared on a variety of inputs. We report here the most significant results.

We have run the above methods on null determinants, small determinants and determinants of random matrices. In the sequel $a$ is said to be random on $b$ bits if $a$ is an integer evenly distributed in the range $-2^{b}+1$ and $2^{b}-1$.

\section{D inputs}

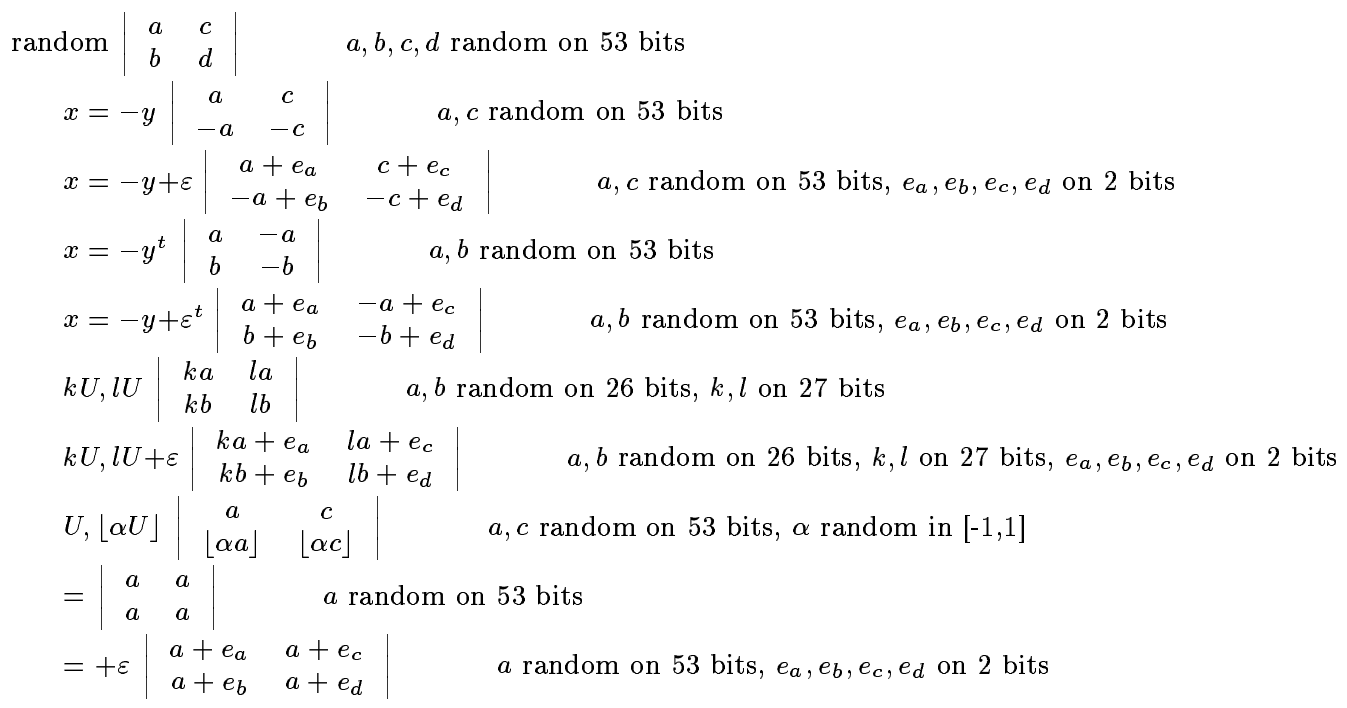


Running time in $\mu$ s.

\begin{tabular}{|l|l||r|r|r|r|r|r|}
\hline \multicolumn{2}{|c|}{ input } & double & quadruple & leda-integer & LN & standard & lazy \\
\hline \multicolumn{1}{|l||}{ random } & 0.58 & 165 & 47 & 2.1 & 2.39 & 0.66 \\
\hline almost & $x=-y+\varepsilon$ & 0.58 & 166 & 50 & 15.7 & 27.60 & 0.64 \\
\cline { 2 - 8 } & $x=-y+\varepsilon^{t}$ & 0.56 & 164 & 47 & 15.6 & 3.49 & 1.41 \\
\cline { 2 - 8 } & $k U, l U+\varepsilon$ & 0.52 & 152 & 47 & 15.9 & 24.91 & 0.66 \\
\cline { 2 - 8 } & $U,\lfloor U\rfloor$ & 0.58 & 159 & 47 & 15.5 & 31.66 & 2.79 \\
\cline { 2 - 8 } & $=+\varepsilon$ & 0.47 & 162 & 48 & 15.8 & 3.08 & 1.18 \\
\hline null & $k U, l U$ & 0.49 & 148 & 47 & 15.9 & 26.26 & 32.10 \\
\cline { 2 - 8 } & $=$ & 0.52 & 160 & 48 & 16.1 & 4.24 & 5.02 \\
\cline { 2 - 8 } & $x=-y$ & 0.54 & 163 & 48 & 16.3 & 53.18 & 64.89 \\
\cline { 2 - 8 } & $x=-y^{t}$ & 0.52 & 164 & 48 & 16.2 & 4.33 & 5.10 \\
\hline
\end{tabular}

\section{D inputs}

$$
\begin{aligned}
& \text { random }\left|\begin{array}{ccc}
a & d & g \\
b & e & h \\
c & f & i
\end{array}\right| \quad a, b, c, d, e, f, g, h, i \text { random on } 51 \text { bits } \\
& x+y+z=0 \mid \begin{array}{ccc|}
a & d & g \\
b & e & h \\
-a-b & -d-e & -g-h
\end{array} \quad a, b, d, e, g, h \text { random on } 51 \text { bits } \\
& x+y+z=0+\varepsilon \mid \begin{array}{ccc|c}
a+e_{a} & d+e_{d} & g+e_{g} & a, b, d, e, g, h \text { random on } 51 \text { bits, } \\
b+e_{b} & e+e_{e} & h+e_{h}
\end{array}
\end{aligned}
$$

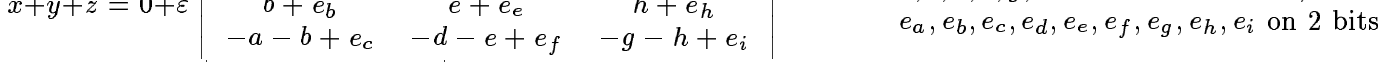

$$
\begin{aligned}
& x+y+z=0^{t}\left|\begin{array}{ccc}
a & d & -a-d \\
b & e & -b-e \\
c & f & -c-f
\end{array}\right| \quad a, b, c, d, e, f \text { random on } 51 \text { bits }
\end{aligned}
$$

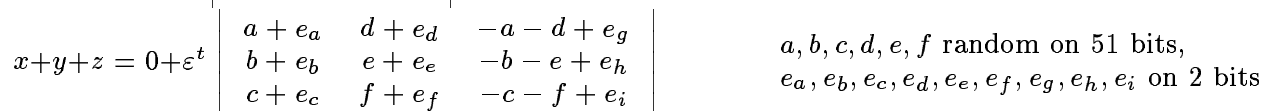

$$
\begin{aligned}
& k U, l V, m U+n V \mid \begin{array}{lll}
k a & l d & m a+n d \\
k b & l e & m b+n e \\
k c & l f & m c+l f
\end{array} \quad a, b, c, d, e, f, m, n \text { random on } 25 \text { bits, } k, l \text { on } 26 \text { bits } \\
& k U, l V, m U+n V+\varepsilon \mid \begin{array}{ccc|c}
k a+e_{a} & l d+e_{d} & m a+n d+e_{g} & a, b, c, d, e, f, m, n \text { random on } 25 \text { bits } \\
k b+e_{b} & l e+e_{e} & m b+n e+e_{h} & k, l \text { on } 26 \text { bits, } \\
k c+e_{c} & l f+e_{f} & m c+l f+e_{i} & e_{a}, e_{b}, e_{c}, e_{d}, e_{e}, e_{f}, e_{g}, e_{h}, e_{i} \text { on } 2 \text { bits }
\end{array} \\
& U, V,\lfloor\alpha U+\beta V\rfloor \mid \begin{array}{ccc|c}
a & d & g & \\
b & e & h & a, b, d, e, g, h \text { random on } 51 \text { bits, } \\
\lfloor\alpha a+\beta b\rfloor & \lfloor\alpha d+\beta e\rfloor & \lfloor\alpha g+\beta h\rfloor & \alpha, \beta \text { random in }[-1 / 2,1 / 2]
\end{array} \\
& =\left|\begin{array}{lll}
a & a & a \\
a & a & a \\
a & a & a
\end{array}\right| \quad a \text { random on } 53 \text { bits } \\
& =+\varepsilon \mid \begin{array}{ccc}
a+e_{a} & d+e_{d} & g+e_{g} \\
b+e_{b} & e+e_{e} & h+e_{h} \\
-a-b+e_{c} & -d-e+e_{f} & -g-h+e_{i}
\end{array} \quad \begin{array}{l}
a \text { random on } 53 \text { bits, } \\
e_{a}, e_{b}, e_{c}, e_{d}, e_{e}, e_{f}, e_{g}, e_{h}, e_{i} \text { on } 2 \text { bits }
\end{array}
\end{aligned}
$$


Running time in $\mu$ s.

\begin{tabular}{|l|l||r|r|r|r|r|}
\hline \multicolumn{2}{|c|}{ input } & double & leda-integer & LN & standard & lazy \\
\hline random & 1.9 & 225 & 3.0 & 14 & 3 \\
\hline \multirow{3}{*}{ almost } & $x+y+z=0+\varepsilon$ & 2.0 & 217 & 65.5 & 310 & 7 \\
\cline { 2 - 6 } & $x+y+z=0+\varepsilon^{t}$ & 2.0 & 222 & 65.4 & 134 & 11 \\
\cline { 2 - 7 } & $k U, l V, m U+n V+\varepsilon$ & 2.0 & 215 & 65.9 & 288 & 15 \\
\cline { 2 - 7 } & $U, V,\lfloor\alpha U+\beta V\rfloor$ & 2.0 & 215 & 65.3 & 303 & 37 \\
\cline { 2 - 7 } & $=+\varepsilon$ & 2.2 & 209 & 71.0 & 164 & 185 \\
\hline null & $k U, l V, m U+n V$ & 2.0 & 219 & 66.9 & 497 & 582 \\
\cline { 2 - 7 } & $=$ & 2.0 & 188 & 77.5 & 18 & 23 \\
\cline { 2 - 7 } & $x+y+z=0$ & 2.0 & 221 & 66.2 & 934 & 910 \\
\cline { 2 - 7 } & $x+y+z=0$ & 2.0 & 219 & 65.7 & 38 & 39 \\
\hline
\end{tabular}

The standard algorithm performs very well on determinants with random entries; even the non lazy version acts as a filter. As this is the situation which is likely to be encountered in practice, our method is believed to run fast in most applications. The null cases are usually very uncommon in practice while the almost null cases are more realistic since nearly degenerate configurations are encountered. In such cases our performance is of the same order of magnitude as the LEDA-integers. Our algorithm is faster than LN in some cases and never exceeds LN by a factor 13 .

\section{Concluding remarks}

We have presented an algorithm that evaluates signs of $2 \times 2$ and $3 \times 3$ determinants with $b$-bit integer entries using only $b$ and $(b+1)$-bit arithmetic respectively. We have also shown how this algorithm can be used in several basic tests in geometric computation. The algorithm has been implemented and compared with the direct computation using several exact methods. Extensive experimental results have been given which demonstrate the efficiency of the algorithm.

An obvious direction for further research is to extend the present work to higher dimensions. The only difficulty there is to generalize Step 2 (Subsection 3.6) which reduces the last component by a constant factor while keeping the other components smaller than $2^{b}$. Such an extension would provide, at least in principle, an extremely general solution to robustness in geometric computation since, by a result of Valiant [Val79], any algebraic expression of size $e$ can be constructively written as an $(e+2) \times(e+2)$ determinant whose entries are either variables or constants.

\section{Acknowledgments}

Jean-Pierre Merlet is acknowledged for supplying to us his interactive drawing preparation system JPdraw . 


\section{References}

[BJMM93] M. Benouamer, P. Jaillon, D. Michelucci, and J.-M. Moreau. A lazy solution to imprecision in computational geometry. In Proc. 5th Canad. Conf. Comput. Geom., pages 73-78, Waterloo, Canada, 1993.

[Cla92] K. L. Clarkson. Safe and effective determinant evaluation. In Proc. 33rd Annu. IEEE Sympos. Found. Comput. Sci., pages 387-395, 1992.

[FM91] S. Fortune and V. Milenkovic. Numerical stability of algorithms for line arrangements. In Proc. 7th Annu. ACM Sympos. Comput. Geom., pages 334-341, 1991.

[For89] S. Fortune. Stable maintenance of point set triangulations in two dimensions. In Proc. 30th Annu. IEEE Sympos. Found. Comput. Sci., pages 494-505, 1989.

[For92] S. Fortune. Numerical stability of algorithms for 2-d Delaunay triangulations and Voronoi diagrams. In Proc. 8th Annu. ACM Sympos. Comput. Geom., pages 83-92, 1992.

[FV93] S. Fortune and C. J. Van Wyk. Efficient exact arithmetic for computational geometry. In Proc. 9th Annu. ACM Sympos. Comput. Geom., pages 163-172, 1993.

[FV94] S. Fortune and C. J. Van Wyk. Ln user manual, 1994.

[GSS89] L. J. Guibas, D. Salesin, and J. Stolfi. Epsilon geometry: building robust algorithms from imprecise computations. In Proc. 5th Annu. ACM Sympos. Comput. Geom., pages 208-217, 1989.

[GY86] D. H. Greene and F. F. Yao. Finite-resolution computational geometry. In Proc. 27th Annu. IEEE Sympos. Found. Comput. Sci., pages 143-152, 1986.

[HHK89] C. M. Hoffmann, J. E. Hopcroft, and M. T. Karasick. Robust set operations on polyhedral solids. IEEE Comput. Graph. Appl., 9(6):50-59, November 1989.

[KLN91] M. Karasick, D. Lieber, and L. R. Nackman. Efficient Delaunay triangulations using rational arithmetic. $A C M$ Trans. Graph., 10:71-91, 1991.

[Mil88a] V. Milenkovic. Verifiable Implementations of Geometric Algorithms using Finite Precision Arithmetic. Phd thesis, Carnegie Mellon University, 1988.

[Mil88b] V. Milenkovic. Verifiable implementations of geometric algorithms using finite precision arithmetic. Artif. Intell., 37:377-401, 1988.

[Mil89] V. Milenkovic. Double precision geometry: a general technique for calculating line and segment intersections using rounded arithmetic. In Proc. 30th Annu. IEEE Sympos. Found. Comput. Sci., pages 500-505, 1989. 
[MN89] K. Mehlhorn and S. Näher. LEDA, a library of efficient data types and algorithms. Report A 04/89, Fachber. Inform., Univ. Saarlandes, Saarbrücken, West Germany, 1989.

[SI88] K. Sugihara and M. Iri. Geometric algorithms in finite-precision arithmetic. Technical Report 88-10, Math. Eng. and Physics Dept., U. of Tokyo, Japan, September 1988.

[Val79] L. Valiant. Completeness classes in algebra. In Proc. 11th Annu. ACM Sympos. Theory Comput., pages 249-261, 1979. 